\title{
Opinion \\ Emerging role of laparoscopy in surgery for pancreatic cancer
}

\section{Opinion}

Pancreatic cancer is one of the most aggressive cancers of the body. Radical surgical resection is the only potentially curable treatment available. The situation is further complicated as only $15-20 \%$ of these patients present with resectable disease. The only curative surgical options available are distal pancreatectomy (DP) for body and tail tumors and pancreaticoduodenectomy (PD) for tumors of head of pancreas. Survival benefit is achieved when done with negative resection margins (R0 resection). Pancreatic surgery is a complex undertaking with higher morbidity and mortality due to the retroperitoneal location, friable structure \& surrounding complex anatomy of the pancreas, intricate dissection involved in pancreatic surgery and complicated anastomoses in cases of PD. Laparoscopy in the management of pancreatic cancer surgery was started in staging and palliation. ${ }^{1}$ The complexity of pancreatic surgery caused slower development of laparoscopic pancreatic resections despite the fact that the first laparoscopic DP and laparoscopic PD were done in early 90 s. $^{2}$ Presently, various laparoscopic procedures shown to be safe and feasible for the management of pancreatic cancer include- staging/ diagnostic laparoscopy with biopsy, palliative biliary $\&$ gastric bypass (Hepatico- and gastro- jejunostomy), DP and PD.

\section{Staging / diagnostic laparoscopy}

This is done in patients with locally advanced disease to detect occult intra-abdominal metastatic disease so as to avoid unnecessary laparotomies. During procedure biopsy of the suspicious lesion and peritoneal cytology can be done by instilling saline into peritoneum. The detection rate of peritoneal metastases ranges from $15-46 \%{ }^{3}$ The addition of laparoscopic ultrasound has improved the yield and accuracy significantly. ${ }^{4}$ The main limitation of laparoscopic staging also includes inability to diagnose local invasion, need of general anaesthesia and additional costs involved. Thus presently the role of laparoscopy in staging of pancreatic cancer is limited.

\section{Laparoscopic distal pancreatectomy (LDP)}

LDP is a simpler procedure as there is limited dissection and no need of anastomosis. The use of hand assisted ports helps in the initial learning curve. Resection margins and lymph node yield are the two most important considerations for curative resection. Many studies have shown similar R0 resection and lymph node yield when compared with open DP. Laparoscopic DP is associated with lesser blood loss, shorter hospital stay, less postoperative pain, faster recovery, less overall costs and better cosmesis. ${ }^{5,6}$ Pancreatic fistula rates were also found to be similar between open and laparoscopic DP when linear stapler were used for pancreatic transection. ${ }^{7}$

\section{Laparoscopic pancreaticoduodenectomy (LPD)}

LPD is the most complex pancreatic operation due to the intricate dissection and need of multiple anastomoses with propensity to major complications. Many reports have shown that it is still feasible though with a steep learning curve and prolonged operative times. Many surgeons use mini-laparotomy and hand ports for creating anastomoses in the initial cases. LPD have been shown to have various advantages
Volume 4 Issue 4 - 2016

\author{
Sundeep Jain, Naresh Jangir \\ Department of GI, HPB \& Minimal access surgery, Fortis \\ hospital, India
}

Correspondence: Sundeep Jain, Department of GI, HPB \& Minimal access surgery, Fortis hospital, A-57, Flat 203, Pearl grand apartment, Shanti Path,Tilaknagar, Jaipur, Rajasthan, India, Tel 0091-9829045733,Email drsundeepjain@yahoo.co.in

Received: December 16, 2015 | Published: April 19, 2016

in terms of lower rates of delayed gastric emptying, less blood loss, less total ICU and hospital stay when compared with open PD. The R0 resection and lymph node yield found similar to that after open $\mathrm{PD} .{ }^{8,9}$ The perioperative morbidity and mortality rates were also found similar to open PD.

Despite the evidence showing feasibility along with similar results, the absence of definitive clinical improvements along with prolonged operative times make its position still controversial. Higher levels of evidence with more controlled trials are needed to reach definitive conclusion.

\section{Robotic laparoscopic pancreatic resections}

Robotic pancreatic resection is still in its infancy. Robotic assistance provides advantages in terms of increased manoeuvrability, precise tissue handling and better visualization in three dimensions. Disadvantages include loss of tactile sensation (as in laparoscopy), equipment setup and maintenance costs and associated learning curve. Promising results have been shown with robotic assisted DP and PD with comparable morbidity and mortality. ${ }^{10}$

\section{Conclusion}

There has been a major development in the role of laparoscopy in the surgical management of pancreatic cancer. The evidence suggests that it is feasible with additional benefits shown with staging laparoscopy and LDP like avoiding unnecessary laparotomies, lesser blood loss, shorter hospital stay, reduced postoperative pain and faster recovery. However the applicability of laparoscopic approach in cancer is limited when compared to open method due to the associated technical complexity, steep learning curve and higher operative times especially in LPD. Also the long term data related to oncological outcomes like tumor recurrence and survival are still not well defined. Nonetheless, recent reports on laparoscopic pancreatic surgery for cancer are encouraging and increasingly practised. 


\section{Acknowledgments}

None.

\section{Funding}

None.

\section{Conflicts of interest}

The authors declare that there is no conflict of interest.

\section{References}

1. Pisters PW, Lee JE, Vauthey JN, et al. Laparoscopy in the staging of pancreatic cancer. Br J Surg. 2001;88(3):325-337.

2. Gagner M, Pomp A. Laparoscopic pylorus preserving pancreatoduodenectomy. Surg Endosc. 1995;8(5):408-410.

3. Jimenez RE, Warshaw AL, Rattner DW, et al. Impact of laparoscopic staging in the treatment of pancreatic cancer. Arch Surg. 2000;135(4):409-414.

4. Dulucq JL, Wintringer P, Mahajna A. Laparoscopic pancreaticoduodenectomy for benign and malignant diseases. Surg Endosc. 2006;20(7):1045-1050.
5. Venkat R, Edil BH, Schulick RD, et al. Laparoscopic distal pancreatectomy is associated with significantly less overall morbidity compared to the open technique: a systematic review and meta-analysis. Ann Surg. 2012;255(6):1048-1059.

6. Jusoh AC, Ammori BJ. Laparoscopic versus open distal pancreatectomy: a systematic review of comparative studies. Surg Endosc 26(4):904-913.

7. Kooby DA, Gillespie T, Bentrem D, et al. Left sided pancreatectomy: a multicenter comparison of laparoscopic and open approaches. Ann Surg. 2008;248(3):438-446.

8. Gumbs AA, Rodriguez Rivera AM, Milone L, et al. Laparoscopic pancreatoduodenectomy: a review of 285 published cases. Ann Surg Oncol. 2011;18(5):1335-1341.

9. Asbun HJ, Stauffer JA. Laparoscopic vs open pancreaticoduodenectomy: overall outcomes and severity of complications using the Accordion Severity Grading System. $J$ Am Coll Surg. 2012;215(6):810-819.

10. Zureikat AH, Moser AJ, Boone BA, et al. 250 robotic pancreatic resections: safety and feasibility. Ann Surg. 2013;258(4):554562. 